\title{
ALGUNAS NOTAS ACERCA DE LA REPRESENTACIÓN MEDIANTE PUNTOS DE NÚMEROS, SERIES Y FUNCIONES
}

\author{
BELL, A. \\ Shell Centre for Mathematical Education. Universidad de Nottingham. UK.
}

Presentamos aquí algunas notas como respuesta al artículo de Rico y otros, Sistemas de representación y aprendizaje de estructuras numéricas. Los números y sus operaciones son herramientas conceptuales para describir y manejar aspectos del mundo, y además constituyen un sistema de conceptos y relaciones que contienen pautas que suscitan interés por sí mismas. La utijización plena del sistema numérico implica el conocimiento tanto de las relaciones generales que existen en su seno como de las propiedades de algunos números en particular. Por relaciones generales entiendo las leyes conmutativa, asociativa y distributiva, el carácter inverso de la multiplicación y la división, y relaciones similares tradicionalmente atribuidas al álgebra. Las propiedades de algunos números en particular son aquéllas relacionadas con los números primos y la divisibilidad, números cuadrados y triangulares, ternas pitagóricas, sucesiones generadas por duplicación, etc.

Un interrogante que suscita Ia primera parte del artículo de Rico es el papel de las representaciones mediante puntos en el aprendizaje de estas relaciones. Considerando primero las propiedades de algunos números en particular, es evidente que las diferenciaciones entre números primos y compuestos se relacionan con la posibilidad de representar el número mediante una matriz rectangular de puntos u otros objetos; pero ello no implica un examen más profundo, especialmente si nos restringimos a matrices planas. Por ejemplo, el carácter especial de 60 , como número con múltiples divisores, puede analizarse perfectamente mediante su expresión numérica como producto de números primos, 22.3 .5 , y no se añade gran cosa mostrando un montón de posibles rectángulos. Los conjuntos de números cuadrados y triangulares son quizá los únicos que se benefician de forma significativa mediante la representación por puntos.

Las leyes conmutativas, por otra parte, pueden demostrarse perfectamente utilizando una matriz rectangular de puntos, que aparece, por ejemplo, como $a \times b$, al contar por filas, y como $b \times a$, al contar por columnas. La ley distributiva resulta igualmente fácil, pero la ley asociativa exige tres dimensiones o bien un razonamiento más complicado mediante varios diagramas de conjuntos. Las leyes inversas tienen que deducirse de sus definiciones, pero pueden ilustrarse convenientemente mediante diagramas de puntos.

\section{REPRESENTACIONES MEDIANTE PUNTOS Y SUCESIONES}

El lugar que corresponde a las sucesiones en los programas de matemáticas, creo que depende de: a) su importancia intrínseca como objetos matemáticos; $b$ ) en tanto que ejemplifican tipos de funciones - Iineal, cuadrática, exponencial, etc. $-;$ y $c$ ) que proporcionen un ámbito de problemas en el que se puedan desarrollar ciertas estrategias matemáticas generales muy importantes. Éstas son: a) la expresión de la relación funcional $n \rightarrow f(n)$, por contraste con la relación intra-sucesión $f(n) \rightarrow f(n+1)$; y $b$ ) la conciencia de que para establecer la validez de tal fórmula es necesario trabajar a partir de la ley definitoria de la sucesión, ya que no puede deducirse de la sucesión numérica generada. La diferencia entre resultado plausible y resultado demostrado queda claramente expuesta.

Estos aspectos de las sucesiones corresponden fundamentalmente a las sucesiones numéricas, no a una sucesión de diagramas de puntos, excepto en los casos de números cuadrados y, probablemente, en los triangulares. De hecho, Ias disposiciones de pentágonos y heptágonos regulares y de polígonos regulares de mayor número de lados no pueden definirse sobre tramas cuadradas o isométricas, y las distorsiones necesarias para acomodarlas a tales tramas implican decisiones arbitrarias. Mi modo de abordar este terreno consiste en tratar las sucesiones de puntos como situación inicial, siendo la tarea que hay que realizar la de generar la sucesión numérica y la de hallar y demostrar la fórmula de $f(n)$. 
Voy a mostrar parte del material elegido de acuerdo con estos objetivos. Al hacerlo, ejemplificaré un comentario sobre otra observación de Rico y otros -la de que el concepto de término n-ésimo resulta diff́cil. Si bien estoy de acuerdo con que la tencencia de los estudiantes es a considerar las relaciones «intra-sucesión» como dominantes, hernos visto que es muy factible enseñar la derivación de la relación funcional, "entre-sucesiones», y su inversa, eligiendo desde el principio ejemplos fáciles y adıcuados y concretando la situación al trabajar con una tabla de dos filas, tal como queda evidenciadoe implicado mediante mi expresión intra y entre, respecto a las sucesiones.

\section{SUCESIONES Y FUNCIONES CON ALUM- NOS DE 12 AÑOS}

El conocimiento de diferentes tipos de variaciones funcionales y la preparación necesaria para trabajar con ellas es uno de los elementos más importantes del programa de matemáticas en esta etapa. Por dicho conocimiento quiero dar a entender el saber qué quiere decir función lineal, proporción, ley de los cuadrados inversos y crecimiento o decrecimiento exponencial, así como tener una cierta nocion acerca de cuáles de los anteriores conceptos pueden aplicarse a una situación práctica determinada. La preparación necesaria consiste en poder trabajar con tablas de valores, obtener y utilizar fórmulas y trazar y leer gráficos. Siendo las sucesiones funciones cuyo dominio es el conjunto de los números naturales, proporcionan un campo de ideas accesible en el que muchos de los conocimientos necesarios pueden desarrollarse en una etapa inicial.

\section{Una primera unidad de trabajo pretendía desarrollar:}

- las nociones de sucesión, pauta de diferencias, función y función inversa;

- la familiarización con una tabla de valores y su correspondiente gráfico;

- Ia expresión del término general, estableciendo la distinción entre $n \rightarrow f(n)$ y $f(n) \rightarrow f(n+1)$, expresándolo con palabras y, en tanto sea posible, con formulas;

- distinciones entre sucesiones lineales y de más alto orden, relacionadas con la línea recta y con gráficas curvas, así como con diferencias primeras y diferencias segundas constantes.

Para las situaciones iniciales, Ios estudiantes construían filas de figuras con palillos -cuadrados, triángulos, cruces, modelos de puntos hechos con estaquillas clavadas sobre un plancha (incluyendo polígonos regulares e irregulares). Tras obtener unos primeros términos de cada sucesión contando, saltaban a números grandes y a $n$. Los siguientes esbozos muestran parte del trabajo.
Filas de triángulos unidos

$\begin{array}{llllllllllllllll}\text { Tamaño } & 1 & 2 & 3 & 4 & 5 & 6 & 7 & 8 & 9 & 20 & 30 & 43 & 100 & \mathrm{n} & \mathrm{m}-1 / 2 \\ \text { Palilios } & 3 & 5 & 7 & 9 & 11 & 13 & 15 & 17 & 19 & 41 & 61 & 87 & 201 & \mathrm{n} \times 2+1 & \mathrm{~m}\end{array}$

1. La pauta que he hallado es: $1+2=3 ; 3+4=7$; $2+3=5 ; 4+5=9 \ldots$

2. Para pasar del número de lados al número de palillos, la pauta es $\times 2$ y añadir 1. Por tanto, cuando te dan un número de los de abajo, se quita 1 y se divide por 2 .

La pauta hallada por el estudiante implica tres elementos adyacentes de la tabla y muestra que las pautas que perciben no siempre son las que nosotros consideraríamos como más pertinentes. La segunda observación del estudiante muestra una excelente conexión entre función directa y función inversa.

Al hacer un comentario acerca de una tabla similar de filas de cuadrados unidos, el estudiante dice: «Los números de abajo crecen de 3 en 3, 1 menos que el número de lados», lo cual muestra tanto la preferencia normal por observar la forma en que los números varían dentro de las filas, en vez de entre las filas, como el reconocimiento de la relación entre el crecimiento de los números y la forma en que cambian los diagramas, de uno al siguiente. La conciencia de que no se puede establecer la regla funcional para $n$ a partir solamente de los números generados, sino de que es necesario volver a la regla de construcción de la sucesión, constituye un importante punto de aprendizaje.

\section{OBTENCIÓN DE FÓRMULAS A PARTIR DE SUCESIONES}

El proceso algebraico típico para hallar la expresion de $T_{n}$ utilizando ecuaciones simultáneas para determinar coeficientes provoca a veces ciertas dificultades. Pero pueden allanarse si se experimenta antes con el proceso inverso; es decir, si se utilizan diversas fórmulas para generar sucesiones y si nos familiarizamos con la relación entre el tipo de fórmula y la pauta de las diferencias de la sucesión. Nosotros hernos utilizado el siguiente material:

\section{Lista de formulas}
1. $t(n)=7 n-3$
8. $\mathrm{I}(\mathrm{n})=3^{\prime} 7-0^{\prime} 4 \mathrm{n}$
2. $t(n)=3 n^{2}$
9. $t(n)=2+3 n$
3. $t(n)=10 \times 2 n$
10. $t(n)=4 n$
4. $t(n)=6-2 n$
11. $t(n)=n-5 n^{2}+17$
5. $t(n)=6$
12. $t(n)=(2 \times n)+6$
6. $t(n)=(n+2)(n-1)$
13. $t(n)=n^{2}+4 n-6$
7. $\mathrm{t}(\mathrm{n})=\mathrm{n}^{2}-4 \mathrm{n}$ 
Cada una de las fórmulas anteriores corresponde a una sucesion. Dando a $n$ un valor concreto, por ejemplo, 3 , se puede hallar el 3er. término.

En la formula 4,

$$
t(3)=6-(2 \times 3)=0
$$

por tanto, el tercer término es 0 .

Estudia cada una de las anteriores fórmulas hallando algunos términos de sus sucesiones y examinando sus pautas de variación. Clasificalas y haz un comentario acerca de ellas. Intenta aprender a establecer qué tipo de sucesión vas a obtener solamente observando su fórmula.

Lista de sucesiones

$\begin{array}{ll}\text { S1 } & 2,4,6,8,10 \\ \text { S2 } & 1,4,9,16,25 \\ \text { S3 } & 4,7,10,13,16 \\ \text { S4 } & 4,12,20,28,36 \\ \text { S5 } & 6,24,54,96,150 \\ \text { S6 } & 2,4,8,16,32 \\ \text { S7 } & 4,4,4,4,4 \\ \text { S8 } & 6,16,30,48,70 \\ \text { S9 } & 3,6,9,12,15 \\ \text { S10 } & 95,89,83,77,71 \\ \text { S11 } & 1,9,25,49,81 \\ \text { S12 } & 2,20,38,56,74 \\ \text { S13 } & 3,5,9,15,23 \\ \text { S14 } & 3,6,12,24,48 \\ \text { S15 } & 4,8,14,22,32 \\ \text { S16 } & 10,8,6,4,2 \\ \text { S17 } & 1,3,6,10,15 \\ \text { S18 } & 23,28,33,38,43 \\ \text { S19 } & 5,8,14,26,50 \\ \text { S20 } & 16,13,7,4,2 \\ \text { S21 } & 3,55^{\prime} 2,77^{\prime} 4,9,11^{\prime} 8 \\ \text { S22 } & 8,7,6,5,4 \\ \text { S23 } & 4,16,37,76,139 \\ \text { S24 } & 2,8,18,32,50 \\ \text { S25 } & 57,4,89^{\prime} 7,122,154^{\prime} 3,186^{\prime} 6\end{array}$

En cada una de las sucesiones anteriores se dan los cinco primeros términos.

Estudia cada sucesión. Intenta clasificarlas según su tipo. ¿Puedes prolongar cada una de las sucesiones? Estudia las pautas de las diferencias. Halla las fórmulas para tantas sucesiones como puedas.

Procediendo primero de fórmula a sucesión, se construye un cuerpo experimental de forma que, cuando se plantea el problema inverso, los estudiantes tienen una idea clara de qué tipo de fórmula se trata de encontrar. La búsquueda de los coeficientes correctos adquiere así mayor significado.

\section{LA NECESIDAD DE RELACIONES ARIT- METICAS}

Rico y otros destacan que, por sí misma, la representación decimal de los números de una sucesión puede dar una escasa idea de la relación funcional, y que expresarla mediante relaciones aritméticas puede significar una cierta ayuda. Ello puede exigir un cierto grado de experimentación, tal como se muestra en la siguiente secuencia de trabajo, que puede tener lugar cuando los estudiantes intentan hallar la fórmula del término $n$-ésimo de la sucesión de números triangulares. Los números que se obtienen son:

$$
1,3,6,10,15,21,28, \ldots
$$

y resulta sencillo considerarlos como:

$$
1,1 \times 3,2 \times 3,2 \times 5,3 \times 5,3 \times 7,4 \times 7,
$$

pero la luz solamente se hace cuando se duplica cada término para dar:

$$
2,2 \times 3,4 \times 3,4 \times 5,6 \times 5,6 \times 7,8 \times 7
$$

que se ordena en la forma:

$$
1 \times 2,2 \times 3,3 \times 4,4 \times 5, \ldots
$$

y así sucesivamente.

A los estudiantes les resulta, entonces, sencillo considerar el término n-ésimo de esta sucesión duplicađa como $n(n+1)$

\section{EJEMPLOS MÁS DIFÍCILES}

Un conjunto de diagramas diseñados por John Mason muestra una serie de sucesiones de puntos que presentan ciertas dificultades para identificar el modo de crecimiento, tanto de los diagramas como de los números. Los siguientes muestran números estrella.

Dos maneras de ver el crecimiento en estos casos (como en los dos diagramas de en medio), a saber, como hexágonos con triángulos en los lados y como una disposición de paralelogramos crecientes que gira, conducen a las siguientes sucesiones:

a) $1,1+6(2), 1+6(2)+6(4), 1+(6(2)+6(4)+6(6), \ldots$

b) $1,1+6\left(1^{\prime} 2\right), 1+6\left(2^{\prime} 3\right)+6\left(3^{\prime} 4\right), \ldots$

que resultan coherentes al observar que la primera tiene como $(n+I)$-ésimo término $l+I 2 T n$, donde $T n$ es el $n$-ésimo número triangular; mientras que la segunda tiene $l+6 n(n+l)$.

\section{NOTA}

Este artículo ha sido traducido del inglés por $J$. Tortella. 
Figura 1

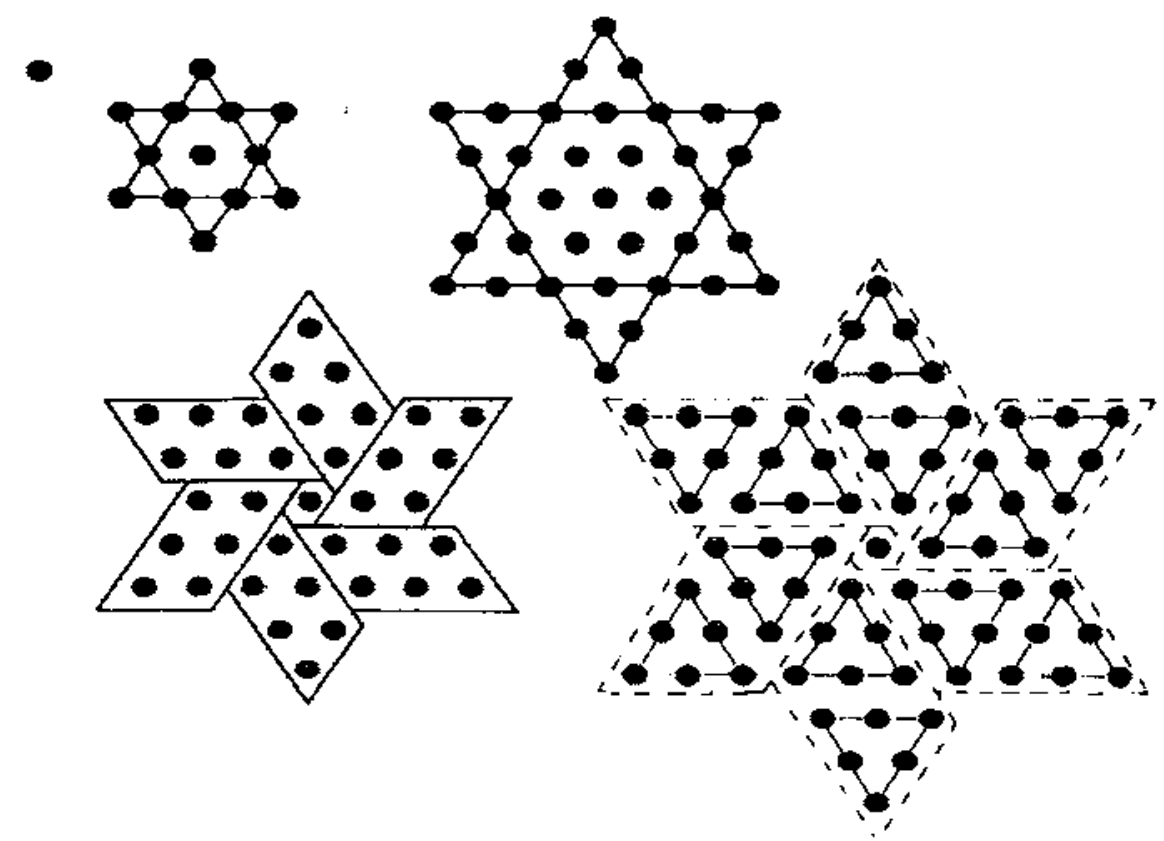

\section{REFERENCIAS BIBLIOGRÁFICAS}

BELL, A., ROOKE, D. y WIGLEY, A. (1978). Journey into Mathematics. Teachers Guide 1. Shell Centre, Universidad de Nottingham.

MASON, J., sin fecha. Polygonal Dot Sequences. 8 posters. Centre for Mathematical Education. Open University, MiIton Keynes.

RICO, L., CASTRO, E. y ROMERO, I. (1996). The role of representation systems in the learning of numerical structures, en Puig. L. y Gutiérrez, A. (eds.) (1996). Proceedings of the 20th Conference of the International Group for the Psychology of Mathematics Education, Vol. 1, pp. 87-102. 